\title{
On Stability Delay Bounds of Simple Input-delayed Linear and Non-linear Systems: Computational Results
}

\author{
Elbrous M. Jafarov
}

Istanbul Technical University, Faculty of Aeronautics and Astronautics, 34469, Maslak, Sariyer, Istanbul, Turkey

\begin{abstract}
This paper deals with the problem of delay size stability analysis of single input-delayed linear and nonlinear systems. Conventional reduction, reduction linked by sliding mode, and linear memoryless control approaches are used for simple input-delayed systems to obtain the stability conditions. Several first order examples are investigated systematically to demonstrate the capabilities and limitations of the advanced stability analysis techniques including Lyapunov-Krasovskii functionals, Newton-Leibniz formula, and a newly addressed Lagrange mean value theorem. Numerical comparative results show the usefulness and effectiveness of the advanced delay size analysis techniques proposed in this paper.
\end{abstract}

Keywords: Input-delayed systems, reduction method, Lagrange mean value theorem, sliding mode control, Lyapunov-Krasovskii functional.

\section{Introduction}

Time-delay effect is frequently encountered in many engineering systems. Delay effect complicates the analysis and design of control systems. A major problem in the analysis and design of time-delayed systems is related to delay-dependent stability conditions and stabilization using linear or discontinuous state feedback with or without memory. Delay-dependent stability and stabilization conditions provided an upper bound of time-delay size, which ensures the asymptotic stability of the system.

As we known, there are three basic conventional control design methods for input-delayed systems: Smith predictor ${ }^{[1]}$, reduction ${ }^{[2]}$ and memoryless control approaches ${ }^{[3,4]}$. Reduction approach linked by variable structure control was used in [5]. Recently, variable structure control ${ }^{[6-8]}$ has also been used for stabilization of time-delay systems.

Recent advances in time-delay systems were presented in $[9-14]$. Several new improved delay-dependent stability and robust stability conditions for time-invariant and uncertain time-delay systems were developed by using standard and augmented Lyapunov-Krasovskii functionals ${ }^{[15-22]}$.

It is clear that the usage of various control design methods may lead to different stability conditions, which may affect the size of the upper bound of the time-delay. From this point of view, conventional reduction, reduction linked by sliding mode, linear memoryless control approaches are used for simple input-delayed systems in this paper. Also, some new stability conditions are obtained and compared numerically.

In this paper, the Lagrange mean value theorem is introduced for the first time to the stability analysis of input delayed systems. This theorem allows expressing the timedelayed control input as a combination of a delay-free input and its derivative. Then, a set of sufficient conditions for the stability are obtained by using Lyapunov-Krasovskii functionals. Numerical comparative results show the usefulness and effectiveness of our advanced analysis techniques.

This paper is organized as follows. In Section 2, delaydependent stability analysis of simple input-delayed linear and nonlinear systems by using classical reduction method and reduction method linked by sliding mode is investigated systematically with several examples. Section 3 presents the conventional memoryless control approaches for simple input-delayed systems. Delay-dependent stability conditions are obtained by using various versions of LyapunovKrasovskii functional candidates, Newton-Leibniz and Lagrange mean value theorem analysis techniques. In Section 4 , the Lagrange mean value theorem approach is introduced for the stability analysis of input-delayed systems and its extension to multivariable systems. Here, some appropriate analysis techniques are advanced. The obtained upper bound of delay size is compared with some existing results. In Section 5, the obtained computational comparison results for numerical examples of single input-delayed systems are analyzed. Finally, conclusions are given in Section 6 .

\section{Reduction method approaches}

Comparative delay-dependent stability analysis of simple input-delayed linear and nonlinear systems with several examples is investigated systematically. Some useful results are presented. The Lagrange mean value theorem is introduced and appropriate analysis techniques are advanced.

Let us consider the following single input-delayed system of the form:

$$
\begin{aligned}
& \dot{x}(t)=A x(t)+b u(t-h), t \geqslant 0 \\
& u(t)=\phi(t),-h \leqslant t \leqslant 0
\end{aligned}
$$

where $x(t)$ is the available $n$-state vector, $u(t)$ is the scalar control input, $A$ is the constant real $n \times n$ matrix, $b$ is the constant $n$-vector, $\phi(t)$ is a known initial control function in $[-h, 0]$, and $h>0$ is a constant time-delay. 


\subsection{Reduction method}

Consider the following input-delayed system:

$$
\dot{x}(t)=A x(t)+b_{0} u(t)+b_{1} u(t-h)
$$

where $b_{0}$ and $b_{1}$ are known vectors, if $b_{0}=0$, then (2) reduces to (1).

By using a linear state transformation ${ }^{[2]}$, we can obtain

$$
z(t)=x(t)+\int_{t-h}^{t} \mathrm{e}^{A(t-h-\theta)} b_{1} u(\theta) \mathrm{d} \theta .
$$

And (2) reduces to the delay-free system as

$$
\dot{z}(t)=A z(t)+\left(b_{0}+\mathrm{e}^{-A h} b_{1}\right) u(t)=A z(t)+\bar{b} u(t)
$$

where $\bar{b}=b_{0}+\mathrm{e}^{-A h} b_{1}$.

The reduced system (4) can be stabilized by using the controller

$$
u(t)=-k^{\mathrm{T}} z(t)
$$

where $k$ is a gain vector.

Choose a conventional Lyapunov function candidate as

$$
V(z(t))=z^{\mathrm{T}}(t) P z(t)
$$

where $P$ is a positive definite symmetric matrix.

Then,

$$
\dot{V}=-z^{\mathrm{T}}(t) Q z(t)<0
$$

if the following Lyapunov matrix equation holds:

$$
P\left(A-\bar{b} k^{\mathrm{T}}\right)+\left(A-\bar{b} k^{\mathrm{T}}\right)^{\mathrm{T}} P=-Q<0
$$

where $Q$ is a positive definite symmetric matrix.

In order to compute the maximum upper bound $\bar{h}$ in $0<h \leqslant \bar{h}$, let us consider the following simple example.

Example 1. Consider the first order input-delayed system

$$
\dot{x}(t)=-a x(t)-b u(t-h)
$$

where $a$ and $b$ are some constant scalars.

By using a linear state transformation

$$
z(t)=x(t)-\int_{t-h}^{t} \mathrm{e}^{-a(t-h-\theta)} b u(\theta) \mathrm{d} \theta
$$

the input-delayed system (9) reduces to

$$
\dot{z}(t)=-a z(t)-\mathrm{e}^{a h} b u(t)
$$

which can be stabilized by the linear delayed controller

$$
u(t)=-k z(t) .
$$

Choose a conventional Lyapunov function as

$$
V=\frac{1}{2} z^{2}(t)
$$

Then,

$$
\dot{V}=-\left(a-\mathrm{e}^{a h} b k\right) z^{2}(t) \leqslant-\left(a-\mathrm{e}^{a h}|b k|\right) z^{2}(t)<0
$$

if

$$
a>\mathrm{e}^{a h}|b k|>0 .
$$

Hence, the upper bound can be obtained from (15) as

$$
\bar{h}=\frac{\ln \frac{a}{|b k|}}{a} .
$$

Thus, the transformed system (11) is asymptotically stable for any $0<h<\bar{h}$ with the maximum upper bound $\bar{h}$ of (16).

\subsection{Reduction method linked by sliding mode}

Now, let us demonstrate how this idea can be used for the control of the single input-delayed system (2) with matched external disturbances:

$$
\dot{x}(t)=A x(t)+b_{0} u(t)+b_{1} u(t-h)+f(t)
$$

where $f(t)$ is an unknown but bounded external disturbance. We assume that it is matched by the control:

$$
\begin{aligned}
& f(t)=\bar{b} \bar{f}(t) \\
& |\bar{f}(t)| \leqslant f_{0}
\end{aligned}
$$

$\bar{f}(t)$ is a scalar function, $f_{0}$ is a scalar constant.

By using the reduction method, the input-delayed system (17) can be transformed into delay-free system

$$
\dot{z}(t)=A z(t)+\bar{b} u(t)+\bar{b} \bar{f}(t) .
$$

Then, we can utilize the following simple conventional sliding mode control

$$
\begin{aligned}
& u(t)=-k^{\mathrm{T}} z(t)-\delta \operatorname{sgn}(s(t)) \\
& s(t)=c^{\mathrm{T}} z(t)
\end{aligned}
$$

where $s(t)$ is the switching function, $c$ is a design $n$-vector, $k$ is a state feedback gain vector, and $\delta$ is a relay gain scalar to be determined. The sufficient conditions for the existence of sliding mode are formulated in Theorem 1.

Theorem 1. Suppose that the matching condition (18) holds. Then an asymptotically stable sliding mode exists on the sliding manifold $s(t)=0$ defined for the transformed system (19) driven by variable structure controller (20) if the following conditions are satisfied:

$$
\begin{aligned}
& c^{\mathrm{T}}\left(A-\bar{b} k^{\mathrm{T}}\right)=\lambda c^{\mathrm{T}} \\
& c^{\mathrm{T}} \bar{b}\left(\delta-f_{0}\right)>0
\end{aligned}
$$

where $\lambda$ is one of the negative eigenvalues of the stable closed loop system matrix $\left(A-\bar{b} k^{\mathrm{T}}\right)$.

Proof. Choose a conventional Lyapunov function

$$
V(s(t))=\frac{1}{2} s^{2}(t) .
$$

Then,

$$
\begin{aligned}
& \dot{V}=s(t) \dot{s}(t)= \\
& s(t) c^{\mathrm{T}}\left(A-\bar{b} k^{\mathrm{T}}\right) z(t)-c^{\mathrm{T}} \bar{b} \delta|s(t)|+c^{\mathrm{T}} \bar{b} \bar{f}(t) s(t) \leqslant \\
& \lambda s^{2}(t)-c^{\mathrm{T}} \bar{b}\left(\delta-f_{o}\right)|s(t)|<-c^{\mathrm{T}} \bar{b}\left(\delta-f_{0}\right)|s(t)|<0
\end{aligned}
$$


if conditions (22) and (23) hold.

In order to compute the maximum upper bound $\bar{h}, 0<$ $h<\bar{h}$, let us consider the following simple example.

Example 2. Again consider a simple input-delayed system (9), which is transformed to (11).

Define a classical relay controller with the switching function as

$$
\begin{aligned}
& u(t)=-\delta \operatorname{sgn}(s(t)) \\
& s(t)=c z(t)
\end{aligned}
$$

where $\delta$ and $c$ are some design constants.

The closed-loop system can be written as

$$
\dot{z}(t)=-a z(t)+\mathrm{e}^{a h} b \delta \operatorname{sgn}(s(t)) .
$$

The sliding condition for (28) is given by

$$
\begin{aligned}
& s(t) \dot{s}(t)<-\left(a-\mathrm{e}^{a h}|c b \delta|\right) s^{2}(t)<0 \\
& \text { if } \quad a>\mathrm{e}^{a h}|c b \delta|>0 .
\end{aligned}
$$

Hence, the maximum upper bound $\bar{h}$ can be obtained as

$$
\bar{h}=\frac{\ln \frac{a}{|c b \delta|}}{a} .
$$

And (28) is asymptotically stable for any $0<h<\bar{h}$ with the upper bound (31).

\section{Linear memoryless control approach}

In this section, conventional memoryless control approaches for simple input-delayed systems are investigated. The delay-dependent stability conditions are obtained by using various versions of Lyapunov-Krasovskii functionals, Newton-Leibniz and Lagrange mean value theorem analysis techniques. From these conditions, the upper bound of time-delays is obtained and compared through a classical test example.

Consider again a first order input-delayed system (9) with memoryless control action

$$
u(t)=-k x(t)
$$

where $k$ is a gain constant.

For the purpose of comparison through the well studied classical test example, let $k=-1$. Then, (9) reduces to the classical first order system, which will be briefly remembered from [10] and extended to another type of functional.

\subsection{Classical test example}

Example 3. The first order system is given by

$$
\begin{aligned}
& \dot{x}(t)=-a x(t)-b x(t-h), t \geqslant 0 \\
& x(t)=\varphi(t),-h \leqslant t \leqslant 0
\end{aligned}
$$

where $a$ and $b$ are known constants, $h>0$ is a constant time-delay, and $\varphi(t)$ is a known function in $-h \leqslant t \leqslant 0$.

Choose a conventional Lyapunov-Krasovskii functional as

$$
V(x(t), x(t-h))=\frac{1}{2} x^{2}(t)+\mu \int_{t-h}^{t} x^{2}(\theta) \mathrm{d} \theta
$$

where $\mu$ is a positive scalar to be selected.

Then, the time-derivative of (34) along (33) is given by

$$
\dot{V}=-\left[\begin{array}{c}
x(t) \\
x(t-h)
\end{array}\right]^{\mathrm{T}}\left[\begin{array}{cc}
a-\mu & \frac{1}{2} b \\
\frac{1}{2} b & \mu
\end{array}\right]\left[\begin{array}{c}
x(t) \\
x(t-h)
\end{array}\right]
$$

Hence, $\dot{V}$ is negative definite if and only if $|H|=$ $(a-\mu) \mu-\frac{1}{4} b^{2}>0$, which gets a maximum value if $\frac{\partial|H|}{\partial \mu}=a-2 \mu=0$ or $\mu=\frac{a}{2}$. Then, $\max |H|=\frac{a^{2}-b^{2}}{4}>0$, from which the stability conditions are obtained as

$$
\begin{aligned}
& a+b>0, \quad a-b>0 \\
& \text { or }-a<b<a \text { or } 0<|b|<a .
\end{aligned}
$$

Note that $\lambda_{\min }(H)=\frac{a-b}{2}>0$ and $\lambda_{\max }(H)=\frac{a+b}{2}>0$, from which the same stability conditions can be obtained as

$$
a-b>0 \text { and } a+b>0 .
$$

Thus, (33) is delay-independent asymptotically stable if condition (36) holds. The only problem here may occur if $b>|a|$. In this case, the maximal critical value of $\bar{h}$ can be exactly found by using the frequency-domain approach

$$
\bar{h}=\frac{\arccos \left(-\frac{a}{b}\right)}{\sqrt{b^{2}-a^{2}}} .
$$

The delay-dependent stability condition by using conventional memoryless control is given in the following example.

Example 4. Again consider a simple time-delay system (33). Choose the augmented Lyapunov-Krasovskii functional as

$$
V=\frac{1}{2} x^{2}(t)+\mu \int_{t-h}^{t} x^{2}(\theta) \mathrm{d} \theta+\rho \int_{-h}^{0} \int_{t+\beta}^{t} x^{2}(\gamma) \mathrm{d} \gamma \mathrm{d} \beta
$$

where $\mu$ and $\rho$ are some positive scalars.

Then,

$$
\begin{gathered}
\dot{V}=-\left[\begin{array}{c}
x(t) \\
x(t-h)
\end{array}\right]^{\mathrm{T}}\left[\begin{array}{cc}
a-\mu-\rho h & \frac{1}{2} b \\
\frac{1}{2} b & \mu
\end{array}\right] \times \\
{\left[\begin{array}{c}
x(t) \\
x(t-h)
\end{array}\right]-\rho \int_{t-h}^{t} x^{2}(\beta) \mathrm{d} \beta<0}
\end{gathered}
$$

if the condition

$$
|H|=(a-\mu-\rho h) \mu-\frac{1}{4} b^{2}>0
$$

with $a-\mu-\rho h>0, \mu>0, \rho>0$.

As seen from (41), if $h=\frac{a-\mu}{\rho}$, and $H \leqslant 0$, system (33) with functional (39) becomes unstable. Therefore, the stability condition and region depend on the form of the selected functional and the size of delay.

Now, we can find the upper bound $\bar{h}$ in a similar way to the one in Example 3 as

$$
\bar{h}=\frac{a^{2}-b^{2}}{b^{2}} \quad \text { with } \quad|b|<a
$$


and $\rho=\mu=\frac{a}{2(1+h)}$.

If $\rho=0$ is substituted into (40) then the delay-dependent conditions (41) and (42) reduce to the delay-independent case.

Now let us consider the Newton-Leibniz formula approach.

\subsection{Newton-Leibniz formula approach}

Example 5. Again consider a simple time delay system (33).

Substituting the Newton-Leibniz formula

$$
x(t-h)=x(t)-\int_{t-h}^{t} \dot{x}(\theta) \mathrm{d} \theta
$$

into (33), we can obtain

$\dot{x}(t)=-(a+b) x(t)-a b \int_{t-h}^{t} x(\theta) \mathrm{d} \theta-b^{2} \int_{t-h}^{t} x(\theta-h) \mathrm{d} \theta$.

Construct an augmented Lyapunov-Krasovskii functional

$$
\begin{aligned}
V= & \frac{1}{2} x^{2}(t)+\mu \int_{-h}^{0} \int_{t}+\theta^{t} x^{2}(\beta) \mathrm{d} \beta \mathrm{d} \theta+ \\
& \rho \int_{-h}^{0} \int_{t+\theta-h}^{t} x^{2}(\beta) \mathrm{d} \beta \mathrm{d} \theta+\gamma \int_{t-h}^{t} x^{2}(\theta) \mathrm{d} \theta
\end{aligned}
$$

where $\mu, \rho$, and $\gamma$ are some positive scalars. Then,

$$
\begin{aligned}
& \dot{V}=x(t) \dot{x}(t)+\mu h x^{2}(t)-\mu \int_{t-h}^{t} x^{2}(\theta) \mathrm{d} \theta+\rho h x^{2}(t)- \\
& \rho \int_{t-h}^{t} x^{2}(\theta-h) \mathrm{d} \theta+\gamma x^{2}(t)-\gamma x^{2}(t-h)= \\
& -(a+b-\gamma) x^{2}(t)-a b x(t) \int_{t-h}^{t} x(\theta) \mathrm{d} \theta- \\
& b^{2} x(t) \int_{t-h}^{t} x(\theta-h) \mathrm{d} \theta+\mu h x^{2}(t)-\mu \int_{t-h}^{t} x^{2}(\theta) \mathrm{d} \theta+ \\
& \rho h x^{2}(t)-\rho \int_{t-h}^{t} x^{2}(\theta-h) \mathrm{d} \theta-\gamma x^{2}(t-h) .
\end{aligned}
$$

For any $h>0$, the Noldus inequality holds:

$$
\begin{aligned}
& h \int_{t-h}^{t} x^{2}(\theta) \mathrm{d} \theta \geqslant\left[\int_{t-h}^{t} x(\theta) \mathrm{d} \theta\right]^{2} \\
& h \int_{t-h}^{t} x^{2}(\theta-h) \mathrm{d} \theta \geqslant\left[\int_{t-h}^{t} x(\theta-h) \mathrm{d} \theta\right]^{2} .
\end{aligned}
$$

Therefore, (46) reduces to

$$
\begin{aligned}
& \dot{V} \leqslant-[(a+b-\gamma)-\rho h-\mu h] x^{2}(t)- \\
& a b x(t) \int_{t-h}^{t} x^{2}(\theta) \mathrm{d} \theta-\gamma x^{2}(t-h)-b^{2} x(t) \\
& \int_{t-h}^{t} x^{2}(\theta-h) \mathrm{d} \theta-\frac{1}{h} \mu\left[\int_{t-h}^{t} x(\theta) \mathrm{d} \theta\right]^{2}- \\
& \frac{1}{h} \rho\left[\int_{t-h}^{t} x(\theta-h) \mathrm{d} \theta\right]^{2}=-\left[\begin{array}{c}
\int_{t-h}^{t} x(t) \\
\int_{t-h}^{t} x(\theta-h) \mathrm{d} \theta \\
x(t-h)
\end{array}\right]^{\mathrm{T}} \times \\
& {\left[\begin{array}{cccc}
a+b-\gamma-\rho h-\mu h & \frac{1}{2} a b & \frac{1}{2} b^{2} & 0 \\
\frac{1}{2} a b & \frac{1}{h} \mu & 0 & 0 \\
\frac{1}{2} b^{2} & 0 & \frac{1}{h} \rho & 0 \\
0 & 0 & 0 & \gamma
\end{array}\right] \times} \\
& {\left[\begin{array}{c}
\int_{t-h}^{t} x(t) \\
\int_{t-h}^{t} x(\theta-h) \mathrm{d} \theta \\
x(t-h)
\end{array}\right]<0}
\end{aligned}
$$

Hence $\dot{V}<0$, if the matrix $H>0$ in (49) is positive definite.

The upper bound $\bar{h}$ can be found numerically from the condition $\lambda_{\min }(H)<0$.

The same problem can be analyzed by using the Lagrange mean value theorem, which is going to be introduced as follows.

\section{Lagrange mean value theorem ap- proach}

Example 6. Again consider the first order system (33). In this case, we will use the Lagrange mean value theorem. Remembering that the Lagrange mean value theorem ${ }^{[23]}$ is stated as

$$
\frac{f(b)-f(a)}{b-a}=f^{\prime}(\xi), a<\xi<b
$$

where $f(x)$ is continuous at every point of the closed interval $[a, b]$ and differentiable at every point of its interior $(a$ $b)$. Then, delay term can be expressed as

$$
x(t-h)=x(t)-h \dot{x}(\theta)
$$

where $\theta$ is a point in the interval $t-h<\theta<t$.

Substituting (51) into (33), we have

$$
\dot{x}(t)=-(a+b) x(t)-a b h x(\theta)-b^{2} h x(\theta-h) .
$$

Note that, (44) obtained by using Newton-Leibniz formula also reduces to (52) after applying the mean value theorem 
to definite integrals ${ }^{[23]}$ :

$$
\frac{\int_{a}^{b} f(x) \mathrm{d} x}{(b-a)}=f(\xi)
$$

where $a \leqslant \xi \leqslant b$, and $f(x)$ is continuous in the closed interval $[a, b]$.

Therefore, according to (53),

$$
\begin{aligned}
& \int_{t-h}^{t} x(\theta) \mathrm{d} \theta=h x(\theta) \\
& \int_{t-h}^{t} x(\theta-h) \mathrm{d} \theta=h x(\theta-h)
\end{aligned}
$$

where $\theta$ is some point in the interval $t-h \leqslant \theta \leqslant t$.

Substituting (54) into (44), we have the same transformed system as in (52).

Now, we can proceed to the analysis of the system (52). First, we need to make Assumption 1.

Assumption 1. The time-delay parameter $\theta(t)$ given by (51) is in general a time-dependent function with domain $t-h(t)<\theta(t)<t$. Then, we assume that the rate of a change of this function satisfies

$$
0<1-\eta \leqslant \dot{\theta}(t) \leqslant 1 \text { and } \dot{h}(t) \leqslant \eta<1
$$

where $\eta$ is a known scalar satisfying $\dot{h}(t) \leqslant \eta<1^{[14,16]}$. Note that if $h(t)=$ const and $\eta=0$, then $\dot{\theta}(t)=1$ is obvious from (55).

Choose an augmented Lyapunov-Krasovskii functional as

$$
\begin{aligned}
V= & \frac{1}{2} x^{2}(t)+\alpha \int_{\theta-h}^{\theta} x^{2}(\xi) \mathrm{d} \xi+\beta \int_{\theta}^{t} x^{2}(\zeta) \mathrm{d} \zeta+ \\
& \gamma \int_{t-h}^{t} x^{2}(\psi) \mathrm{d} \psi
\end{aligned}
$$

where $\alpha, \beta$ and $\gamma$ are some positive scalars.

The time-derivative of (56) along (52) is given by

$$
\begin{aligned}
\dot{V}= & -(a+b) x^{2}(t)-a b h x(t) x(\theta)-b^{2} h x(t) x(\theta-h)+ \\
& \beta x^{2}(t)+\dot{\theta}(t) \alpha\left[x^{2}(\theta)-x^{2}(\theta-h)\right]- \\
& \dot{\theta}(t) \beta x^{2}(\theta)+\gamma x^{2}(t)-\gamma x^{2}(t-h) .
\end{aligned}
$$

Since $(55)$ holds and $-\dot{\theta}(t) \leqslant-(1-\eta)$, it follows that

$$
\begin{aligned}
& \dot{V} \leqslant-\left[\begin{array}{cccc}
x(t) & x(\theta) & x(\theta-h) & x(t-h)
\end{array}\right] \\
& {\left[\begin{array}{cccc}
a+b-\beta-\gamma & \frac{1}{2} a b \bar{h} & \frac{1}{2} b^{2} \bar{h} & 0 \\
\frac{1}{2} a b \bar{h} & -\alpha+(1-\eta) \beta & 0 & 0 \\
\frac{1}{2} b^{2} \bar{h} & 0 & \alpha(1-\eta) & 0 \\
0 & 0 & 0 & \gamma
\end{array}\right] \times} \\
& {\left[\begin{array}{c}
x(t) \\
x(\theta) \\
x(\theta-h) \\
x(t-h)
\end{array}\right]<0}
\end{aligned}
$$

if $H>0$.

The upper bound $\bar{h}$ can be found numerically from $H \geqslant 0$ if one of the eigenvalues equals to zero.

\subsection{Extension: Multivariable time-delay system}

The results obtained in Example 6 can be extended to the following multivariable time-delay system:

$$
\dot{x}(t)=A x(t)+A_{1} x(t-h)
$$

where $A$ and $A_{1}$ are given $n \times n$ matrices.

Substituting (51) into (58), we have

$$
\dot{x}(t)=\left(A+A_{1}\right) x(t)-h A_{1} A x(\theta)-h A_{1}^{2} x(\theta-h) .
$$

Theorem 2. Suppose that Assumption 1 holds. Then the transformed time-delay system (59) is delay-dependent asymptotically stable if there exist some positive definite symmetric matrices $P, R, S$ and $T$ such that the following conditions are satisfied:

$H=\left[\begin{array}{cccc}Q & \bar{h} D & \bar{h} C & 0 \\ \bar{h} D^{\mathrm{T}} & R-(1-\eta) S & 0 & 0 \\ \bar{h} C^{\mathrm{T}} & 0 & -(1-\eta) R & 0 \\ 0 & 0 & 0 & -(1-\eta) T\end{array}\right]<0$

where $D=P A_{1} A$ and $C=P A_{1}^{2}$. The following two conditions have to be satisfied for negative definiteness of $H$ in defined in (60):

$$
\begin{aligned}
& Q=P\left(A+A_{1}\right)+\left(A+A_{1}\right)^{\mathrm{T}} P+S+T<0 \\
& 0<R<(1-\eta) S .
\end{aligned}
$$

Proof. By choosing an augmented Lyapunov-Krasovskii functional

$$
\begin{aligned}
& V=x^{\mathrm{T}}(t) P x(t)+ \\
& \int_{\theta-h}^{\theta} x^{\mathrm{T}}(\zeta) R x(\zeta) \mathrm{d} \zeta+\int_{\theta}^{t} x^{\mathrm{T}}(\xi) S x(\xi) \mathrm{d} \xi+ \\
& \int_{t-h}^{t} x^{\mathrm{T}}(\varphi) T x(\varphi) \mathrm{d} \varphi
\end{aligned}
$$


the time derivative of (63) along (59) can be evaluated as

$$
\begin{aligned}
& \dot{V}=x^{\mathrm{T}}(t) P \dot{x}(t)+\dot{x}^{\mathrm{T}}(t) P x(t)+ \\
& \dot{\theta}(t) x^{\mathrm{T}}(\theta) R x(\theta)-\dot{\theta}(t) x^{\mathrm{T}}(\theta-h) R x(\theta-h)+ \\
& x^{\mathrm{T}}(t) S x(t)-\dot{\theta}(t) x^{\mathrm{T}}(\theta) S x(\theta)+x^{\mathrm{T}}(t) T x(t)- \\
& x^{\mathrm{T}}(t-h) T x(t-h)= \\
& x^{\mathrm{T}}(t)\left(P\left(A+A_{1}\right)+\left(A+A_{1}\right)^{\mathrm{T}} P\right) x(t)-2 h x^{\mathrm{T}}(t) P A_{1} A x(\theta)- \\
& 2 h x^{\mathrm{T}}(t) P\left(A_{1}\right)^{2} x(\theta-h)+\dot{\theta}(t) x^{\mathrm{T}}(\theta)(R-S) x(\theta)- \\
& \dot{\theta}(t) x^{\mathrm{T}}(\theta-h) R x(\theta-h)+x^{\mathrm{T}}(t)(S+T) x(t)- \\
& x^{\mathrm{T}}(t-h) T x(t-h) \leqslant \\
& x^{\mathrm{T}}(t)\left(P\left(A+A_{1}\right)+\left(A+A_{1}\right)^{\mathrm{T}} P\right) x(t)-2 h x^{\mathrm{T}}(t) P A_{1} A x(\theta)- \\
& 2 h x^{\mathrm{T}}(t) P\left(A_{1}\right)^{2} x(\theta-h)+x^{\mathrm{T}}(\theta) R x(\theta)- \\
& (1-\eta) x^{\mathrm{T}}(\theta) S x(\theta)-(1-\eta) x^{\mathrm{T}}(\theta-h) R x(\theta-h)- \\
& x^{\mathrm{T}}(t-h) T x(t-h) \leqslant\left[\begin{array}{c}
x(t) \\
x(\theta) \\
x(\theta-h) \\
x(t-h)
\end{array}\right]^{\mathrm{T}} \times \\
& {\left[\begin{array}{cccc}
Q & \bar{h} D & \bar{h} C & 0 \\
\bar{h} D^{\mathrm{T}} & R-(1-\eta) S & 0 & 0 \\
\bar{h} C^{\mathrm{T}} & 0 & -(1-\eta) R & 0 \\
0 & 0 & 0 & -T
\end{array}\right] \times} \\
& {\left[\begin{array}{c}
x(t) \\
x(\theta) \\
x(\theta-h) \\
x(t-h)
\end{array}\right]<0, \text { if } H<0 .}
\end{aligned}
$$

Example 7. Consider the linear time-delay system (58) with parameters taken from [17]:

$$
A=\left[\begin{array}{cc}
-2 & 0 \\
0 & -0.9
\end{array}\right], A_{1}=\left[\begin{array}{cc}
-1 & 0 \\
-1 & -1
\end{array}\right]
$$

The maximum allowable bounds of the time-delay computed from condition (60) using LMI control toolbox gives the solution $h_{\max }=1.1074$, where $\eta=0.001, H$ is shown in the end of this page, and

$$
\begin{aligned}
\operatorname{eig}(H)= & (1.0 \mathrm{E}+004) \times[-1.0380-0.3392-0.0054-0.0009 \\
- & 0.0002-0.0001-0.0000-0.0000]^{\mathrm{T}} \\
P & =\left[\begin{array}{c}
2039.7-18.7 \\
-18.70 .8
\end{array}\right] \\
R & =\left[\begin{array}{c}
1614.1-15.2 \\
-15.20 .7
\end{array}\right] \\
S & =\left[\begin{array}{c}
5625.4-34.3 \\
-34.31 .5
\end{array}\right] \\
T & =\left[\begin{array}{c}
26.7728-1.1711 \\
-1.17110 .0525
\end{array}\right]
\end{aligned}
$$

if $\eta=0.00001, h_{\max }=1.1081$.

By considering the well known existing results from [17] where $h_{\max }=0.8571$, from [18] where $h_{\max }=$ 0.9999 , from $[20,21]$ where $h_{\max }=1.0$, it can be concluded that the obtained result $h_{\max }=1.1081$ of the proposed Theorem 2 is comparable with and even better than the existing results of $[17-21]$. Note that, in Theorem 2, the recently highly improved methods with augmented Lyapunov-Krasovskii functionals such as Park inequality ${ }^{[19]}, \mathrm{Xu}$ and Lam relaxation ${ }^{[15]}$ or descriptor system approach ${ }^{[22]}$ are not used. Moreover, the condition (60) is simpler than the ones of existing approaches, and the functionals (63) are standard. However, the largest available time-delay bound obtained in the literature for the numerical example (65) is $h_{\max }=4.47^{[15,19,22]}$.

\section{Computational comparison analysis}

The maximum upper bound of delay size can be found numerically from the stability conditions presented above in a systematical way by using various control approaches. The computational results for the same first order system with the same numerical parameters are given in Table 1. In the left column of this table, the names of appropriate methods with obtained conditions are given whereas the maximum allowable delay bounds are presented in the right column. As seen from Table 1, all the compared conditions derived in this paper give different but comparable upper delay bounds. Therefore, these conditions and approaches are useful. For example, for the same parameters, NewtonLeibniz formula gives $h_{\max }=1.8685$ while the Lagrange

$$
\begin{aligned}
& H=(1.0 \mathrm{E}+003) \times \\
& {\left[\begin{array}{cccccccc}
-6.5486 & 0.0552 & 4.4761 & -0.0186 & 2.2174 & -0.0207 & 0 & 0 \\
0.0552 & -0.0016 & -0.0395 & 0.0008 & -0.0188 & 0.0009 & 0 & 0 \\
4.4761 & -0.0395 & -4.0057 & 0.0191 & 0 & 0 & 0 & 0 \\
-0.0186 & 0.0008 & 0.0191 & -0.0009 & 0 & 0 & 0 & 0 \\
2.2174 & -0.0188 & 0 & 0 & -3.2249 & 0.0304 & 0 & 0 \\
-0.0207 & 0.0009 & 0 & 0 & 0.0304 & -0.0014 & 0 & 0 \\
0 & 0 & 0 & 0 & 0 & 0 & -0.0535 & 0.0023 \\
0 & 0 & 0 & 0 & 0 & 0 & 0.0023 & -0.0001
\end{array}\right]}
\end{aligned}
$$


Table 1 Maximum upper bounds of delay size

\begin{tabular}{|c|c|}
\hline Methods & Maximum allowable delay \\
\hline \multicolumn{2}{|l|}{ Classical test example Lyapunov-Krasovskii method: } \\
\hline 1) Condition (36) $a=0.3, b=0.2, b<a$ & Delay-independent $h_{\max }=\infty$ \\
\hline 2) Nyquist criterion, condition(38) $a=0.3, b=0.6, b>a$ & $h_{\max }=\frac{2 \pi}{3}=2.093$ \\
\hline Reduction method, condition (16) $a=0.3, b=0.2, k=-1$ & $h_{\max }=1.3515$ \\
\hline \multicolumn{2}{|l|}{ Reduction method linked by sliding mode, Condition (31) } \\
\hline$a=0.3, b=0.2, \delta=0.9, c=0.9$ & $h_{\max }=6.1728$ \\
\hline \multicolumn{2}{|l|}{ Memoryless control approach, Lyapunov-Krasovskii method: } \\
\hline 1) Condition (41) $a=0.3, b=0.2$ & $h_{\max }=1$ if $\mu=0.1, \rho=0.1$ \\
\hline 2) Condition (42) $a=0.3, b=0.2$ & $h_{\max }=1.25$ if $\mu=0.1, \rho=0.0001 ; h_{\max }=0.5$ if $\mu=0.1, \rho=0.1$ \\
\hline \multicolumn{2}{|l|}{ Newton-Leibniz formula condition (49): } \\
\hline 1) $a=0.3, b=0.2, b<a$ & $h_{\max }=1.8685$ if $\mu=0.1 ; h_{\max }=2.65$ if $\mu=0.01 \rho=0.01, \gamma=0.102$ \\
\hline 2) $a=0.3, b=0.6, b>a$ & $h_{\max }=1.319$ if $\mu=0.1 ; h_{\max }=0.196$ if $\mu=0.01, \rho=0.01, \gamma=0.102$ \\
\hline \multicolumn{2}{|l|}{ Lagrange mean value theorem, condition (57): } \\
\hline 1) $a=0.3, b=0.2, b<a, \alpha=0.01, \beta=0.01, \gamma=0.102$ & $h_{\max }=1.69$ if $\eta=0.09 ; h_{\max }=1.67$ if $\eta=0.1$ \\
\hline 2) $a=0.3, b=0.2, b<a, \alpha=0.1, \beta=0.1, \gamma=0.102$ & $h_{\max }=5.014$ if $\eta=0.09 ; h_{\max }=4.952$ if $\eta=0.1$ \\
\hline 3) $a=0.3, b=0.6, b>a, \alpha=0.01, \beta=0.01, \gamma=0.102$ & $h_{\max }=0.209$ if $\eta=0.09 ; h_{\max }=0.206$ if $\eta=0.1$ \\
\hline 4) $a=0.3, b=0.6, b>a, \alpha=0.1, \beta=0.1, \gamma=0.102$ & $h_{\max }=1.409$ if $\eta=0.09 ; h_{\max }=1.393$ if $\eta=0.1$ \\
\hline
\end{tabular}

mean value theorem gives $h_{\max }=5.014$. Therefore, the last condition is less conservative than the other ones. Notice that the numerical examples for a first order input delay system, which is systematically investigated in this paper, have not been handled by others yet.

\section{Conclusions}

In this paper, single input-delayed systems have been investigated systematically by using conventional reduction, reduction linked by sliding mode, and linear control design approaches. Delay-dependent stability conditions are obtained by using various types of Lyapunov-Krasovskii functionals, Newton-Leibniz formula, and newly introduced Lagrange mean value theorem analysis techniques. From these conditions, the upper bounds of time-delay are obtained and compared through a classical test example. Comparative numerical results show the usefulness of advanced analysis techniques proposed in the paper. It is believed that the usage of the Lagrange mean value theorem has a great potential for further investigations of input-delayed systems.

\section{References}

[1] O. J. M. Smith. Feedback Control Systems, New York: McGraw-Hill, 1958.

[2] W. H. Kwon, A. E. Pearson. Feedback stabilization of linear systems with delayed control. IEEE Transactions on Automatic Control, vol. 25, no. 2, pp. 266-269, 1980.

[3] J. H. Kim, E. T. Jeung, H. B. Park. Robust control for parameter uncertain delay systems in state and control input. Automatica, vol. 32, no. 9, pp. 1337-1339, 1996.
[4] H. Y. Su, J. Chu, J. C. Wang. A memoryless robust stabilizing controller for a class of uncertain linear time-delay systems. International Journal of Systems Science, vol. 29, no. 2, pp. 191-197, 1998.

[5] Z. Feng, M. Cheng, W. B. Gao. Variable structure control of systems with delays in control. Control and Decision, vol. 8 , no 2, pp. 95-100, 1993. (in Chinese)

[6] K. K. Shyu, J. J. Yan. Robust stability of uncertain timedelay systems and its stabilization by variable structure control. International Journal of Control, vol.57, no.1, pp. 237-246, 1993.

[7] X. Q. Li, R. A. DeCarlo. Robust sliding mode control of uncertain time delay systems. International Journal of Control, vol. 76, no. 13, pp. 1296-1305, 2003.

[8] E. M. Jafarov. Robust sliding mode controllers design techniques for stabilization of multivariable time-delay systems with parameter perturbations and external disturbances. International Journal of Systems Science, vol.36, no.7, pp. 433-444, 2005.

[9] W. Perruquetti, J. P. Barbot. Sliding Mode Control in Engineering, New York: Marcel Dekker, 2002.

[10] J. K. Hale. Functional Differential Equations, Berlin, Heidelberg: Springer-Verlag, 1971.

[11] J. P. Richard. Time-delay systems: An overview of some recent advances and open problems. Automatica, vol. 39, no. 10, pp. 1667-1694, 2003.

[12] S. I. Niculescu, K. Q. Gu. Advances in Time-delay Systems, Berlin: Springer-Verlag, 2004. 
[13] K. Q. Gu, V. L. Kharitonov, J. Chen. Stability of Timedelay Systems, Boston: Birkhauser, 2003.

[14] D. Li, J. -M. Li. Adaptive iterative learning control for nonlinearly parameterized systems with unknown time-varying delay and unknown control direction. International Journal of Automation and Computing, vol. 9, no.6, pp. 578-586, 2012.

[15] S. Y. Xu, J. Lam. Improved delay-dependent stability criteria for time-delay systems. IEEE Transactions on Automatic Control, vol. 50, no. 3, pp. 384-387, 2005.

[16] S. Xu, J. Lam, Y. Zou. Simplified descriptor system approach to delay-dependent stability and performance analyses for time-delay systems. IEE Proceedings: Control Theory and Application, vol. 152, no. 2, pp. 147-151, 2005.

[17] X. Li, C. E. de Souza. Criteria for robust stability and stabilization of uncertain linear systems with state delay. $\mathrm{Au}$ tomatica, vol. 33, no. 9, pp. 1657-1662, 1997.

[18] S. I. Niculescu, A. T. Neto, J. M. Dion, L. Dugard. Delaydependent stability of linear systems with delayed state: An LMI approach. In Proceedings of the 34th IEEE Conference on Decision and Control, IEEE, New Orleans, LA, USA, vol. 2, pp. 1495-1496, 1995.

[19] P. G. Park. A delay-dependent stability criterion for systems with uncertain time-invariant delays. IEEE Transactions on Automatic Control, vol.44, no.4, pp.876-877, 1999.

[20] J. H. Kim. Delay and its time-derivative dependent robust stability of time-delayed linear systems with uncertainty.
IEEE Transactions on Automatic Control, vol. 46, no. 5, pp. 789-792, 2001.

[21] D. Yue, S. Won. An improvement on delay and its timederivative dependent robust stability of time-delayed linear systems with uncertainty. IEEE Transactions on Automatic Control, vol. 47, no. 2, pp. 407-408, 2002.

[22] E. Fridman, U. Shaked. A descriptor system approach to $H_{\infty}$ control of linear time-delay systems. IEEE Transactions on Automatic Control, vol. 47, no. 2, pp. 253-270, 2002.

[23] J. Stewart. Calculus, Early Transcendentals, 7th ed., Canada: Brookd/Cole, 2012.

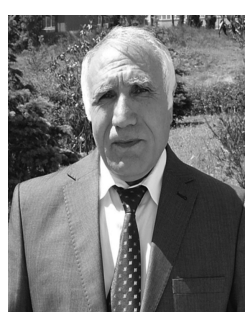

Elbrous M. Jafarov received his M. Sc degree in automation and control engineering from State Oil Academy, Azerbaijan in 1969, his Ph. D. degree from Research Institute of Automation (NIPINefte KhimAutomat-TsNIIK Automation) and Institute of Cybernetics of Azerbaijan Science Academy, Azerbaijan in 1972, and his D. Sc.(Eng.) degree in systems and control engineering from Institute of Control Sciences of USSR University (MIEM-LETI), in 1981. He has been a professor in Istanbul Technical University since 1996. He was a visiting professor at Beijing Aeronautical and Astronautical University (BUAA), in 1993, where he was awarded a China BUAA Advisory Professor Certificate.

His research interests include control theory, sliding mode control, time-delay systems, flight control, robust control, and optimal control.

E-mail: cafer@itu edu.tr 